\title{
Research on Application of Digital Technology in Anhui Intangible Cultural Heritage Popularization Design-- Take the manufacture technology of Huangshan Maofeng tea as an example
}

\author{
Linsen $\mathrm{Li}^{1 *}$, Muyu $\mathrm{Li}^{2}$, Ting Deng ${ }^{3}$ \\ ${ }^{1}$ Academy of Art and Design, Anhui University of Technology, Ma'anshan, Anhui,243032, China
}

\begin{abstract}
Taking the manufacture technology of Huangshan Maofeng intangible cultural heritage in Anhui as a study case, this paper discussed the application of digital technology in expressing intangible cultural heritage science popularization, and proposed protection and inheritance strategies of intangible cultural heritage in Anhui based on information visualization and science popularization. By constructing digital communication model of intangible cultural heritage, it popularized knowledge of intangible cultural and the production process, making the intangible cultural heritage closer to ordinary people's life, and offering a new protection and communication approach for intangible culture heritage, which applies to the development in this time.
\end{abstract}

\section{Introduction}

Intangible cultural heritage, as a typical carrier of traditional life style with cultural characteristics, is the product of historical and cultural accumulation. It was formed through continuous accumulation from history development, with obvious regional characteristics. Intangible cultural heritage has unique historical and cultural values, national spiritual values, and arts and crafts values. In terms of long-term development, it has cultural sharing values, cultural identity values and cultural confidence values. Based on the development of digital technology, information visualization science popularization design offers intangible culture a new communication media, of which, the combination will come up with new forms. Therefore, intangible cultural heritages are different from cultural relics and other tangible materials, they transform with culture development and environment change. Not all intangible cultural heritages can remain in its' original state, their development need to be inherited, merged and innovated by us.

\section{Anhui Intangible Cultural Heritage}

\subsection{Anhui Intangible Cultural Heritage}

Anhui culture consists of four cultural circles: Huizhou culture, Wanjiang culture, Luzhou culture and Huaihe culture. Geographically, Anhui is divided into southern, central and northern Anhui by Yangtze River and Huaihe River. The overall distribution in Anhui shows more in the south and less in the north, forming a situation of "one central area and two core areas".One center area means ancient Huizhou, while the two core areas are Anqing and $\mathrm{Lu}$ 'an. Thus, the distribution of intangible cultural heritage is influenced by culture. The ancient Huizhou culture has influenced Huizhou people, and deriving multiple intangible cultural heritages by retaining and inheriting.

According to Chinese national intangible characteristics system, intangible cultural heritage is divided into ten classes; Anhui has obtained all of the ten categories (figure 1). From the figure, traditional skills and traditional drama show bigger numbers, despite the small quantity of traditional medicine, sports, recreational and acrobatic class project, in terms of number, the rest projects are relatively parallel. Among the ten categories, traditional handicraft has outstanding development, especially from Anhui, showing a concentrated distribution situation. Among them, the tea making technique accounts for $19 \%$ of the traditional handicraft items. Ancient Huizhou is a traditional tea production area. The author takes Huangshan Maofeng Tea as an example to discuss its popular science visual design strategy. 


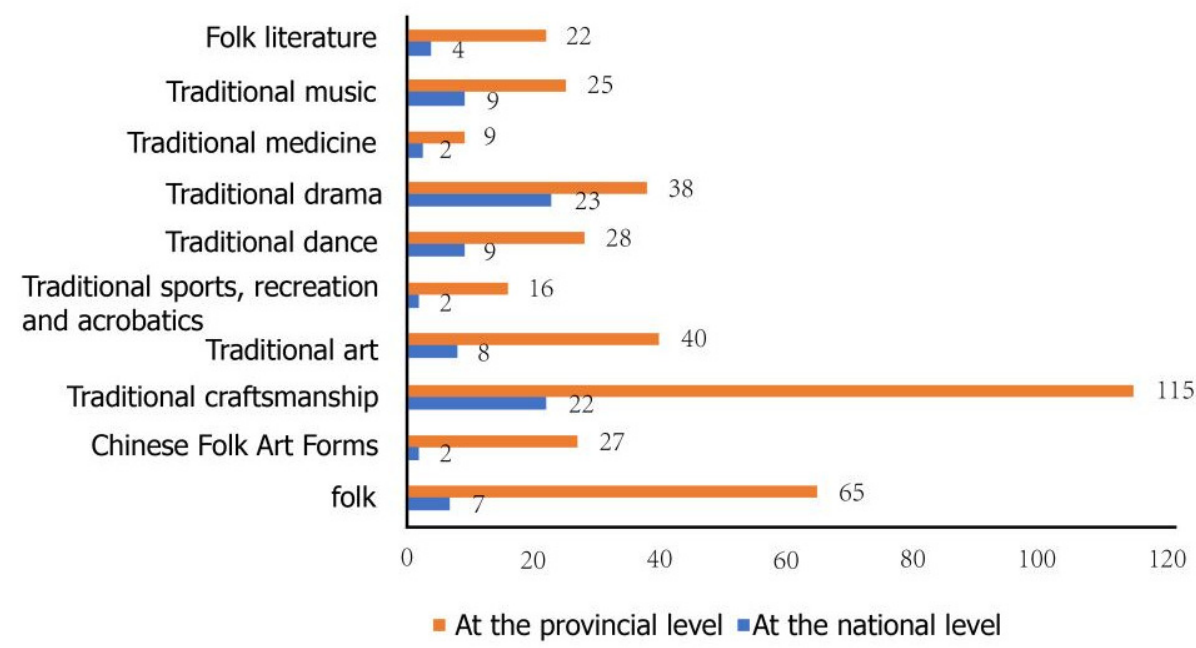

Fig1. The number of intangible cultural Heritage items of different types

\subsection{Huangshan Maofeng}

The prosperity of Huizhou culture is inseparable from the rise of Huizhou merchants. Tea is one of the four proprietors of Huizhou merchants and has an indelible relationship with Huizhou economy. The tea culture industry of Huizhou intangible cultural heritage includes traditional tea making techniques, tea ceremony and tea art, etc. (Table 1). Huangshan city is a gathering area of traditional tea production techniques, the suitable environment, soil conditions are the reasons lead to tea products varieties, which has extremely high historical values. However, with time pasts, some of the "living state" intangible cultural heritages rarely get attention, the values are easily be ignored, leading to the loss of intangible cultural heritages.

Table1. Intangible cultural heritage project resources

\begin{tabular}{cl}
\hline Intangible cultural heritage projects & \multicolumn{1}{c}{ Resources in detail } \\
\hline \multirow{2}{*}{ Tea customs, Tea Gifts } & Life tea customs: three bath tea, full moon tea, first year tea, \\
& enlightenment tea, master tea \\
& Wedding tea customs: four-time tea, three tea six ceremony \\
& Three teas: Jujube and chestnut tea, Egg tea, Maofeng green tea \\
Tea ceremony, Tea art & Rich room tea, intellectuals tea, Buddhist tea, Taoist tea, farm tea \\
& National intangible cultural heritage: Qimen black tea production \\
& skills, green tea production skills (Huangshan Maofeng, Taiping \\
Traditional tea making techniques & Houkui) \\
& Provincial intangible cultural heritage: green tea production \\
& techniques (Tunxi green tea, Songluo tea), Dinggu Dafang \\
& production techniques \\
& Tea proverbs, tea riddles, tea rhymes, tea dances, tea plays, tea \\
& poems, tea books, tea antithetical couplet, tea stories \\
\hline
\end{tabular}

Huangshan Maofeng originated in the Reign of Emperor Guangxu from Qing Dynasty (around 1875 AD). At first, it was named "Maofeng" due to its shape like a peak. After that, it was titled with the production place as "Huangshan Maofeng". According to the records of "Shexian County · Products", "Maofeng, young tea leaves, Xinyuan in the south, tiaoling in the East, Huangshan in the north, which are all producing areas. The tea from Huangshan area is the most famous, of which, the color and fragrance are beyond the tea from others mountains". The writing tells the growth environment of Huangshan Maofeng tea is an indispensable aspect to make it famous. The excellent ecological environment, fertile soil and excellent quality of tea trees are the key factors for the growth of Huangshan Maofeng tea, soaking from Huizhou culture and laying the foundation for the development and expansion of Huangshan Maofeng.

Huangshan Maofeng has a unique classification, divided into prime class, first class, second class, and third class. Prime class and first class are seen as famous tea (Table 2). The prime class Huangshan Maofeng tea is boutique, shaped like a bird tongue, evenly strong, with obvious pekoe, ivory color, like golden fish leaves, when brewing, it has fog around the top, nice smell, fresh taste, clear liquid. The tea leaves are plump, has tender yellow at the bottom [2]. 
Table2. Classification of Huangshan Maofeng Tea

\begin{tabular}{cl}
\hline Level & \multicolumn{1}{c}{ Form } \\
\hline Prime level & Developing one bud and one leaf \\
First level & Developed one bud and one leaf, developing two leaves and one bud \\
Second level & Developed one bud and two leaves, developed one bud and three leaves \\
Third level & Developed one bud and one leaf, two leaves, three leaves \\
\hline
\end{tabular}

\section{Popular science visual design of intangible cultural heritage in converge medium environment}

\subsection{Popularization of Intangible Cultural Heritage- -Production technology of Huangshan Maofeng Tea}

Nowadays, the development of Internet and digital technology has promoted the diversification of media information communication and rapidly penetrated into all fields. Converge media refers to the integration of different information communication media through the Internet [3]. That is to say, the integration of traditional media, new media, new means of communication and new means of produces communication lead to multiple means of communication methods which flowing crossing different media. Science popularization under the environment of converge media breaks the traditional model of science popularization and proposes a new mode of communication, so as to achieve cross-media communication of science popularization information and diversified information dissemination to the public.

Popular science means to use easy language, to explain a variety of scientific phenomena and ethical writing. Qian Xuesen pointed out that popular science knowledge includes natural science, but also social science, mathematical science, system science, thinking science, human science, military science, literature and art theory, and behavioral science [4]. Visualized science popularization informatization can be regarded as the reaction of science popularization practice to the informatization context, that is, "informative science popularization". It also reflects the communication and diffusion of scientific technological information in the informatization context, that is, "science popularization of informatization". Science popularization informatization mainly emphasizes online science popularization as the main way and the transformation mode of science popularization, and takes science popularization informatization as a new method of science popularization to link the information society and provide a new way of communication.

Information visualization is to simplify and convert complex information to visual items. Through the integration of text, graphics, symbol elements and colors, to sort and classify the information is in the context of inner connections. Intangible cultural Heritage popularization belongs to the cross field of intangible cultural heritage protection and science popularization, the integration reflected in two aspects: one is the integration of content; the other is the integration of media forms. Therefore, the visualization of popular science information of intangible cultural Heritage needs to sort, classify, collect and integrate. Through the principle of visual design, and to sufficient communication with converge media, it makes the intangible cultural heritage effectively spread in a new way of communication with public attention arouse.

\subsection{Popular science visual structure design of huangshan Maofeng tea production technology}

\subsubsection{Linear structure}

Intangible cultural heritage integrates media design and popularization, shows the history of Huangshan Maofeng tea in the form of linear structure, and better interpreted in the form of pictures and texts (Figure 2). Huangshan tea production can be traced back to the Song Dynasty, jiayou Years, rise in the Ming Dynasty, during the reign of Emperor Guangxu, "Xie Yutai" tea shops, placed in Tangri and Chong Chuan area of Huangshan. Plump buds were picked at high altitude and sold to northeast and North China through fine processing. As tiny white hair above all the tea leave, like a peak bud, the tea was named "Mao Feng", after, titled the production place name for "Huangshan Maofeng".

\begin{tabular}{|c|c|c|c|c|c|}
\hline 187 & 1955 & 1982 & 1993 & 1995 年 & 2008 \\
\hline $\begin{array}{l}\text { Creation of } \\
\text { "Huangshan } \\
\text { Maofeng" }\end{array}$ & $\begin{array}{l}\text { One of the top } \\
\text { ten famous teas } \\
\text { in China }\end{array}$ & $\begin{array}{l}\text { Won the title of } \\
\text { famous tea of } \\
\text { ministry of } \\
\text { Commerce }\end{array}$ & $\begin{array}{c}\text { Cao Xi tea factory } \\
\text { was established in } \\
\text { Cao Xi, the } \\
\text { birthplace of } \\
\text { Huangshan }\end{array}$ & $\begin{array}{c}\text { The trademark } \\
\text { Cao Xi was } \\
\text { registered }\end{array}$ & $\begin{array}{c}\text { Green tea } \\
\text { production } \\
\text { techniques } \\
\text { (Huangshan } \\
\text { Maofeng) as the } \\
\text { second batch of } \\
\text { national } \\
\text { intanaible cultura }\end{array}$ \\
\hline
\end{tabular}

Fig2. Development history of Huangshan Maofeng tea 


\subsubsection{Contrast structure}

Compare the similarities and differences between green tea and black tea making techniques of huizhou intangible cultural heritage traditional techniques, as to analyze the characteristics of each production techniques through contrast and comparison. Though these two intangible techniques belong to the Huangshan region, they have slice differences from each other, green tea production techniques (Huangshan Maofeng tea) belong to the Huangshan city Huizhou area, black tea production techniques (Qimen black tea) belong to the Qimen county. The similarities and differences are showed by comparative structure to analyze the characteristics of Huangshan Maofeng tea production process. The production technology of Huangshan Maofeng is divided into traditional production method and mechanical production method. The traditional production method is divided into picking, grading, water-removing, rolling, baking; Mechanical production methods are divided into water-removing, strip, rolling, drying. The production techniques of Qimen black tea can be divided into primary and refined production, which can also be divided into picking, withering, rolling, fermentation and drying, and then refined for reprocessing, which can be divided into drying, sieving, sorting, baking and spreading. The two techniques are different in picking standards (Figure 3). Huangshan Maofeng tea is divided into prime class, class 1,class 2, class3. Prime class refers to picking before and after Qingming Festival, and class1-3 refers to picking before and after Grain Rain. Qimen black tea, however, has no grade classification, picking before and after Qingming festival and grain rainly with one bud and two leaves, while high-quality Qimen black tea is chosen as one bud and one leaf or tender bud. In addition to the classification, the scientific method of picking huangshan Maofeng should be "leave only the initial leaf".

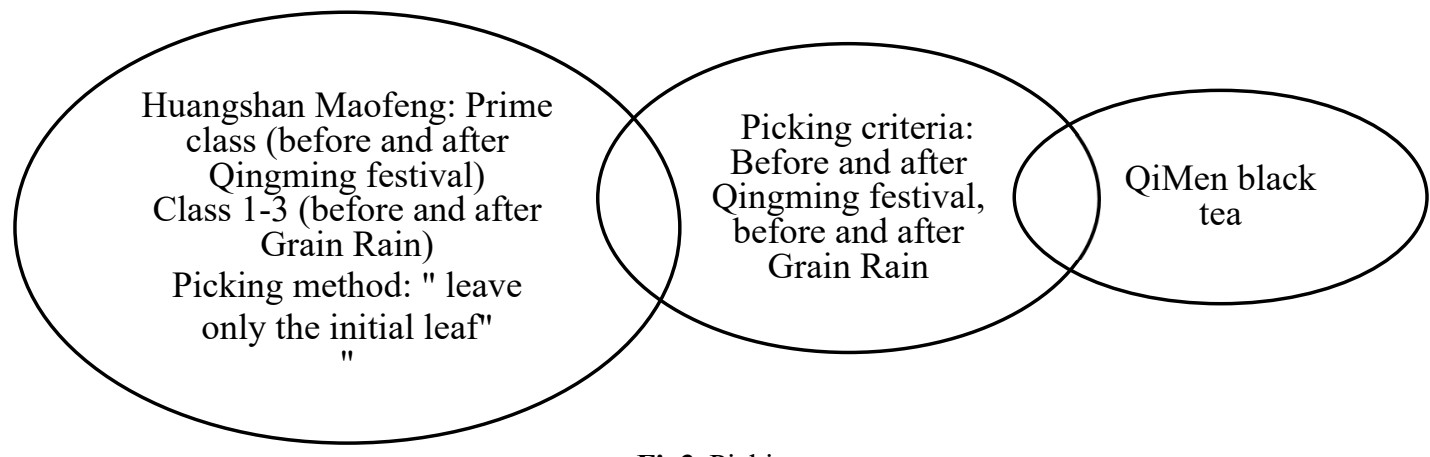

Fig3. Picking

The first process of Huangshan Maofeng tea production process (mainly by hand process) is to remove water, the first process of Qimen black tea production process is withering, they all need to grade before processing, then spread the leaves when pick out the unstandardized leaves, stems and tea fruit. The second process is rolling, these two processes are very similar to ensure the quality of tea leaves. In the third process, the production of Huangshan Maofeng tea is to bake. It is advisable to bake with oven until the water content is $4 \%$ $6 \%$. Remove the impurities and fill the barrel while it is hot, then the traditional production process of Huangshan Maofeng is completed. The production of Qimen black tea needs to be fermented by steam, followed by oven drying. At this point, the initial production process is completed, next, step to the refining process (Figure 4). There are similarities and differences in the production process of the two teas.

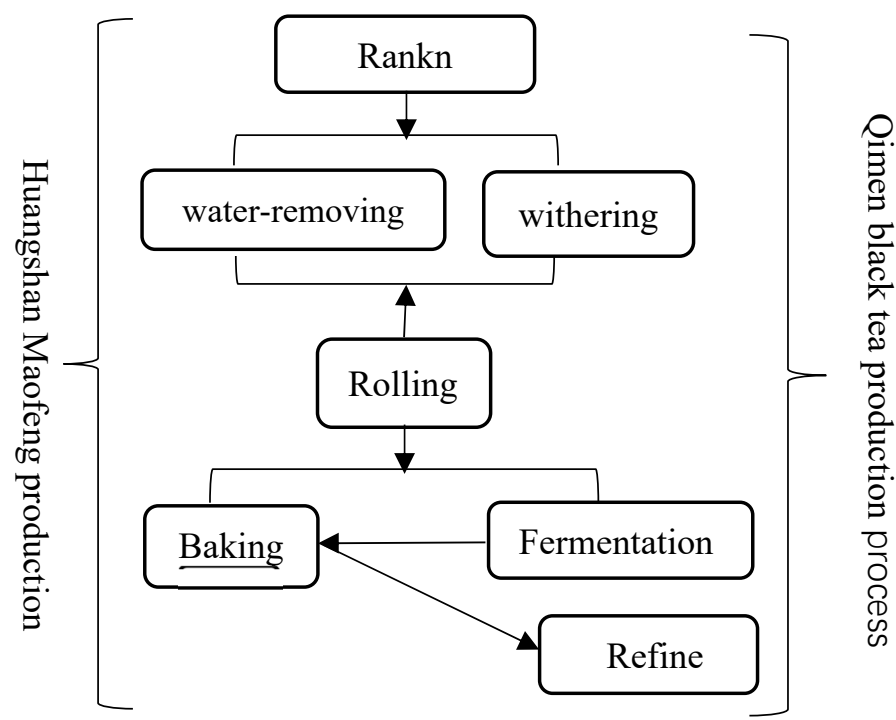

Fig4. Comparison of processing techniques of Huangshan Maofeng tea and Qimen Black tea 


\subsection{Science Popularization design strategy of Huangshan Maofeng production technology}

The production technology of Huangshan Maofeng tea is divided into manual production and mechanical production; the author focuses on the analysis of manual production. As a national intangible heritage, handmaking skills has been gradually separated from the living environment of contemporary people, therefore, protecting and inheriting the traditional hand-making skills is the most priority.

This article aims at popularization, digital protection and inheritance of Huangshan Maofeng tea production process, take informatization design of Huangshan
Maofeng's production process as a core. The research is supported by key information visualization technologies, and undertakes visual design of Huangshan Maofeng's production process. The element to popularizing the production technology of Huangshan Maofeng lies in the way of information communication, and different ways of information communication correspond to different ways of media communication (Table 3). At present, text media and print media still play dominant roles, while with the development of Internet and new media, the single communication effect of traditional media can no longer meet the public's demand for information.

Table3. Information expression of Huangshan Maofeng production technology

\begin{tabular}{ccc}
\hline Information expression mode & Mode of media communication & Media conditions \\
\hline Graphic & Written media, printed media & $\begin{array}{c}\text { Low, high communication } \\
\text { audience }\end{array}$ \\
Audio and video & Television, radio, the Internet & $\begin{array}{c}\text { High, accurate delivery of } \\
\text { content }\end{array}$ \\
Interactive & H5, Virtual reality, Internet of \\
Things & $\begin{array}{c}\text { Relatively higher, Increase } \\
\text { public participation }\end{array}$ \\
\hline
\end{tabular}

The production technology of Huangshan Maofeng tea takes three media communication methods to integrate with each other. With the help of digital technology in different communication methods and conditions from the old and new media, it leads to a process of mutual integration and aggregation as to achieve a new integrated media and produce accurate, vivid information communication.

The establishment of the three ways of informatization expression requires the information construction according to the needs of the audience and the target group. In order to express the production technology of Huangshan Maofeng tea, it is necessary to collect

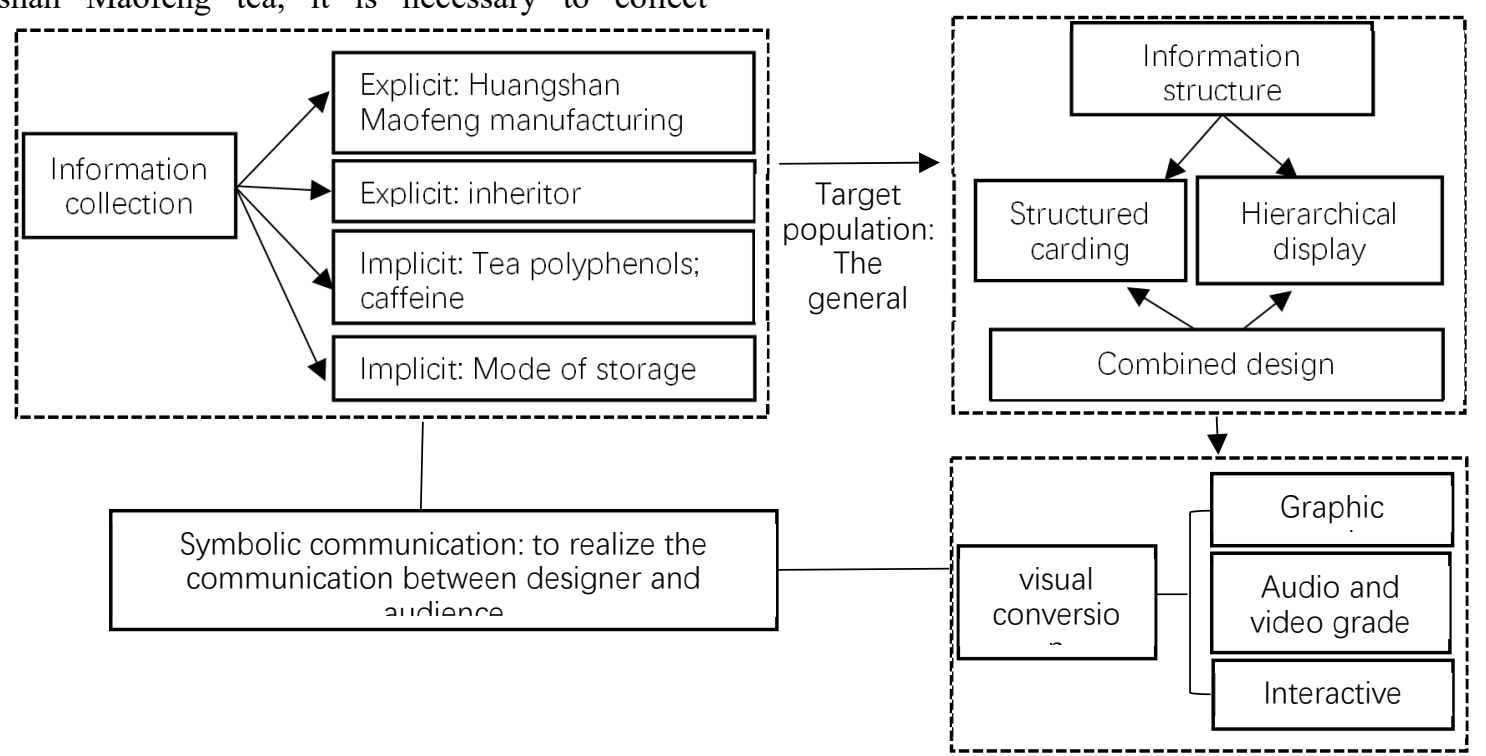

Fig5. Science popularization information construction of Huangshan Maofeng manufacturing Technology

The explicit and implicit information of Huangshan handicraft production is listed and presented. With the combination of Science and technology with culture, it information first, and sort out and classify the collected information. The second step is information structure, which organically arranges the information to achieve effective reading, by expressing the implicit and explicit information. The third step is to complete the visual transformation, take use of three media communication ways to organically integrate communication information. The finally step is to complete the symbol transmission. After the information transformation of the three media, it is a process to encode and decode complex information. The sender and receiver complete the information transmission with symbols as the intermediary (Figure 5).

popularizes the production technology of Huangshan Maofeng tea to the general public at the same time. It also help to explain the chemical composition of Huangshan 
Maofeng tea, as well as the identification, function, teamaking method, taboo notes and storage method.

\section{Conclusions}

Traditional media has its limitations, while, with the development of information technology, the combination of traditional media and new media can be effectively transmitted through various channels, so that the scope of communication is larger and the effect of communication is greatly improved. Through the related technology of science popularization visualization, this research reveals material basis and intrinsic value of Anhui intangible cultural heritage, and helps to develop the visualization design of science popularization of intangible cultural heritage. The way to visualize popular science information of intangible cultural heritage in relevant area can be combined with the fusion of old and new media, in order to provide new information science popularization platform for traditional intangible culture protection and communication. It effectively improves the communication of information visualization design of intangible culture. With the method of culture with science and technology, taking general audience as the core, this research tends to complete science information visualization under the environment of converge media, as to efficiently improve the intangible cultural heritage information communication.

\section{Reference}

1. Peng Jian et al. Spatial and temporal distribution characteristics and influencing factors of intangible cultural heritage in Anhui Province [J]. Journal of Leshan Normal University, 2017, (05): 60-69

2. Tao Dechen. Preliminary knowledge of Huangshan Maofeng Tea [J]. Guizhou tea, 2013, (03): 29-32

3. $\mathrm{Fu}$ Yuhui. Big media industry: from media convergence to industrial convergence [M]. Beijing: China Radio and television press, 2008.

4. Yuan Qinglin. Introduction to popular science [M]. Beijing:China Science and Technology Press, 2002: 20 